\title{
Entertainment Virtual Reality System for Simulation of Spaceflights Over the Surface of the Planet Mars .
}

\author{
Ricardo Olanda \\ Departamento de Informática \\ Universidad de Valencia \\ Vicente Andrés Estellés, $\mathrm{S} / \mathrm{N}$ \\ 46100 Burjassot, Spain \\ Ricardo.Olanda@uv.es
}

\author{
Manolo Pérez \\ Instituto de Robótica \\ Universidad de Valencia \\ Polígono de la Coma, S/N \\ 46980 Paterna, Spain \\ Manolo.Perez@uv.es
}

\author{
Pedro Morillo \\ Departamento de Informática \\ Universidad de Valencia \\ Vicente Andrés Estellés, S/N \\ 46100 Burjassot, Spain \\ Pedro.Morillo@uv.es
}

\author{
Marcos Fernández \\ Departamento de Informática \\ Universidad de Valencia \\ Vicente Andrés Estellés, S/N \\ 46100 Burjassot, Spain \\ Marcos.Fernandez@uv.es
}

\author{
Sergio Casas \\ Instituto de Robótica \\ Universidad de Valencia \\ Polígono de la Coma, S/N \\ 46980 Paterna, Spain \\ scasas@glup.uv.es
}

\begin{abstract}
In recent years Virtual Reality technologies have enabled astronomers to recreate and explore three dimensional structures of the Universe for scientific purposes. Mars, due to its scientific interest, has been the focal point of numerous research projects using these technologies, however, none of these virtual reality tools have been developed specifically for entertainment purposes.

The focus of this paper is to present MarsVR, as an entertainment research project that educates people on the topography and orography of the planet Mars from the perspective of popular science. Some projects have been designed MarsVR for entertainment purposes and include the latest advances in 3D real time applications. However, these applications have underestimated the relevant data necessary for simulating the planet Mars as an interactive virtual environment.
\end{abstract}

\section{Categories and Subject Descriptors}

D.O [Software]: General

\section{General Terms}

Design, Experimentation,Human Factors

\footnotetext{
*Partially supported by the European Commission Network of Excellence INTUITION IST-NMP-1-507248-2
}

Permission to make digital or hard copies of all or part of this work for personal or classroom use is granted without fee provided that copies are not made or distributed for profit or commercial advantage and that copies bear this notice and the full citation on the first page. To copy otherwise, to republish, to post on servers or to redistribute to lists, requires prior specific permission and/or a fee.

VRST'06, November 1-3, 2006, Limassol, Cyprus.

Copyright 2006 ACM 1-59593-321-2/06/0011 ...\$5.00.

\section{Keywords}

Entertainment Virtual Reality, Immersive Visualization Systems, Terrain Representation

\section{INTRODUCTION}

The realistic recreation of interplanetary flights has been one of the most ambitious goals in fields such as astronomy and astrophysics [11]. In the search of mechanisms for representing this type of simulations, Virtual Reality has become the most common and viable option for many different scientists. Virtual Reality not only provides great images, it also gives the user the feeling of navigation and manipulation of the virtual world. Researchers of space sciences have been paying close attention to these technologies.

Systems based on virtual reality technologies are complex to design and implement. However, this complexity becomes more evident when virtual reality systems are developed in the scope of astronomy for two reasons. First, the amount of data (in terms of number of polygons and textures) handled by these applications in real-time exceeds the capacity of personal computers for processing and representing this kind of synthetic environments (planets [31, $32]$, constellations of stars [29, 35], etc). Secondly,this particular limitation causes most three-dimensional re-creations for astronomy purposes possible only by means of high-end, shared memory computers [34]. These applications are usually designed on immersive visualization devices like CAVEs [7], DisplayWalls or Spherical systems [29]. Immersive visualization environments are virtual reality systems where users can view, navigate and/or modify three dimensional models with a first-person perspective. The type of immersion achieved in these complex and expensive systems enable users to behave in the $3 \mathrm{D}$ virtual scene in the same way they would behave in a real environment.

The case of Virtual Reality for entertainment purposes is quite different. The commercial development of entertainment applications based on Virtual Reality technologies appeared as extensions of existing serious applications of flight 
simulation training in the late 1980's [2]. Although these applications received much attention in the early 1990's [15], the cost of the final system was so high that it became unaffordable for commercial purposes. This situation changed when affordable game machines (which included virtual reality technologies) were introduced to the home market [36]. Unlike scientific projects based on Virtual Reality, the simulated environment in virtual reality games was significantly different from reality. Since design of virtual reality games is focused on maximizing the playability while maintaining a significant level of graphic quality, the realistic recreation of represented scenes were not a priority objective. This behavior is evident in the case of the famous game Doom3 (the action happens on the planet Mars) where the graphics performance of the game hides a few astronomy mistakes [14].

In this paper we present an entertainment virtual reality system called MarsVR. The intention of MarsVR is to spread knowledge about the planet Mars with a special focus on the efficiency and implications of geographic visualizations as a key aspect in planetary science education. Even though several systems have recreated the Mars surface using virtual reality techniques [10, 12, 26, 27, 29, 30], to our knowledge no entertainment system has achieved this objective while maintaining the level of realism required for the teaching of astronomy and astrophysics. The research involved in the development of MarsVR project is funded by the regional government of Aragon (Spain) and was motivated to stimulate the interest of students of all ages in the learning of planetary science.

To improve the experience of users in the virtual environment we use the CAVE environment [7, 12] for displaying the surface of Mars. Moreover, we employ a head tracking system to monitor the observer's position as well as a wireless stereoscopic visualization system.

The first version of the MarsVR system has already been presented in two popular science exhibitions. In both demonstrations, MarsVR not only attracted the attention of the general public to the Red Planet, but also allowed us to test the multiplatform capability of our system in terms of both operative systems and immersive visualization hardware support

The rest of the paper is organized as follows: Section 2 refers to related work that has been done in the field of application of Virtual Reality technologies for the teaching of astronomy at all levels. Section 3 describes the proposed system architecture and discusses basic issues for the performed implementations of MarsVR on different hardware platforms. Section 4 shows results obtained by the developed system when it was presented in two different science exhibitions. Finally, Section 5 presents some concluding remarks and the future work to be done.

\section{RELATED WORK}

Historically, the greatest value of Virtual Reality in astronomy is its ability to allow scientists to explore the three dimensional structure of the Universe [19]. The main applications of Virtual Reality developed in this scientific field showed the complexity embedded in 3D graphics applications when planets, stars or galaxies are simulated.

The level of complexity involved in the development of virtual reality applications for astronomy purposes have not impeded research projects that show the real potential of virtual reality techniques when they are applied to the space and planetary sciences [34]. In this sense, the development of new techniques and equipments in the field of Virtual Reality has been quickly adopted and applied with great success in astronomy projects $[12,6,17,24,27,31,32]$. In [32] a three dimensional model of the planet Earth using a third-person navigation can be explored through an HMD (Head Mounted Diplay) device. This device includes stereo visualization and a tracking system for telling the computer the position and orientation of the user's head. Using the planet Earth,recent work demonstrates the most common visualization techniques for the recreation of synthetic terrains [31].

Other efforts have brought the 3D visualization of astronomical data to the general public in digital form. Digital planetaria, such as the Hayden Planetarium in New York and the Denver Science Museum have developed a considerable body of visualization work with astronomical data for entertainment and education purposes. On a lower level, companies and organizations such as Spitz Inc., Sky-Skan, Immersion Institute, Immersion Studios Inc. and the JASON Foundation have developed entertainment and education productions oriented to immersive visualization devices.

The wide diversity of astronomy projects based on Virtual Reality has recreated synthetic scenarios for the Earth $[31,32,6]$, the Moon [17] and the Sun [24]. However, the recreation of planet Mars surface has attracted the interest of researchers from the point of view of Computer Graphics. This interest is derived from the need to represent huge amounts of data obtained from Mars Global Surveyor and Mars Pathfinder space probes. Unlike Mars Global Surveyor (succeeded in entering orbit around Mars in September 1997) the objective of Mars Pathfinder was to land on Mars' surface and to release a 23-pound rover named Sojourner that explored the landing area collecting data and taking images [18]. The recreation of the Pathfinder mission [27], including the Sojourner rover [26], using virtual reality techniques showed unprecedented results in the field of astronomy and validated virtual reality as a powerful cartographic tool.

Taking into account the success of previous works, further contributions extended the scope of virtual reality techniques for cartographic purposes by incorporating new elements in the realistic simulation of the Mars surface. These contributions not only add visual quality to the virtual scenes [3], but also include the use of haptic devices [30] and immersive visualization systems [12] in order to improve the interaction with the virtual environment.

As interactive systems based on Virtual Reality were becoming more accessible to the public, astronomers took advantage of the capabilities provided by these technologies for improving the quality of the teaching in astronomy and astrophysics at different levels. In this sense, a virtual reality system has been presented for the purpose of understanding basic astronomical phenomena such as the reasons for the seasons, lunar and solar eclipses [35]. In this system students can visualize relationships between objects (the Earth, Moon, and Sun) and events (such as eclipses) from different $3 \mathrm{D}$ perspectives. More recently, a paper has shown the successful experiences of a set of undergraduate students when they have studied an introductory planetary geology course on Mars by using an immersive visualization system (CAVE) $[10]$. 
composed of a set of nodes. Figure 2 shows how the architecture of MarsVR is organized as a set of components that are arranged following a layered approach. Each node in the cluster runs a distinct and complete copy of the MarsVR application identified by the Clustering Layer. This top layer is independent of the actual implementation of the graphics features included in MarsVR. Clustering layer achieves the creation of a single system image through use of distributed processing to collect and compute resources together. This software layer is in charge of mimicking the behavior of a single, shared memory computer and showing the system as a single unit to the application developer.

The same encapsulation idea has been applied for the development of the lowest layer in MarsVR called Network Layer. The Network Layer provides a message-passing interface for communicating with the entire cluster. In this instance, this layer is an abstraction of the nodes in the cluster to communicate. The Network Layer offers an interface for exchanging messages with the rest of the nodes of the system as well as maintaining local repositories for building messages and processing them. These characteristics compose the network access function of MarsVR and avoids the low-level access of the Application Layer to the network device drivers.

The Application Layer is the most important layer in the entire model. The Application Layer is built on top of the Network Layer and implements the main functionality of MarsVR. This layer is controlled by a finite state machine (FSM) which accesses the results provided by four software modules in each frame. The finite state machine consists of four states describing the behavior of the $3 \mathrm{D}$ tour through the planet Mars. These steps, corresponding to the stages in the planetary exploration, are described in Section 4 . The modularity introduced into this layer of design not only allows easy future expansion, but also offers the possibility of modifying the application model by adding more software components. The rest of the four software modules controlled by the finite state machine in the Application Layer are called: Synchronization Module, Physics Module, HUD Module and Terrain Generation Module.

Synchronization Module ensures that all the applications running on the cluster nodes begin their execution on the same frame. This module creates a software barrier by sending signals between the cluster nodes. The Synchronization Module uses a master/slave paradigm where each slave sends a signal to the master immediately before swapping the frame buffers. The master is identified through a specific configuration, and the remaining nodes are then identified as slaves. All the slaves then suspend their execution, waiting for the master to send a response signal. The master sends its response immediately before invoking the frame buffer swap operation. Once it has received the response from the master, the slaves perform the frame buffer swap.

Physics Module included in MarsVR is responsible for calculating the position, movement and interaction of the user with the surrounding environment. The complexity of this software module is caused by two aspects. First, the size, density and gravity of the Mars Planet vary significantly from the values measured for the planet Earth. Second, and related to the preceding point, is related to the workload generated by the flight modes included in MarsVR. These two out-of-the-ordinary exploration modes included in the $3 \mathrm{D}$ tour correspond to a spaceflight and a roller coaster ride over the Mars surface. In this sense, the trajectory of the spacecraft is modeled as a cubic spline interpolation. This interpolation uses cubic polynomials to attempt to model a smooth and continuous trajectory which joins a reduced set of points located within the scene [5]. On the other hand, in order to recreate a true roller coaster experience, the roller coaster ride is designed following a realistic physics model described in [33].

HUD Module shows information related to the current position of the user within the virtual scene. This information is located on the upper-right corner of the screen. For each frame, HUD (Head-Up Display) module depicts the current user information following two different criteria. On the one hand, a text panel informs users about information related to the current altitude above sea level, speed and position coordinates (in terms of latitude and longitude). This panel also shows information about the name of the region in which the user is currently located (Mars surface is divided into 30 different regions). On the other hand, the current user position is marked with a point on a small three-dimensional representation of the planet Mars. This small three-dimensional model is located above the surface corresponding to the text panel and is surrounded by the textual information previously described.

Terrain Generation Module (TGM) is one of the main contributions of MarsVR. TGM is really an advanced real-time GIS (Geographic Information System) application. The main objective of TGM is to build and keep a consistent representation of the Mars surface in real time. In order to obtain the maximum visual quality from the original data without sacrificing system performance, TGM includes a multiresolution model of the terrain consisting in a new hierarchial triangulation model based on a non-restricted quadtree representation. Moreover, and depending on the system platform, bumpmapping techniques based on pixel shader have been incorporated into TGM to create advanced lighting effects $[9]$.

\subsection{Planetary Terrain Visualization}

TTraditionally, the real time visualization of various computer generated terrain has become a hot research topic in the field of Computer Graphics. Nowadays, different techniques have been proposed to manage massive textured terrain datasets without burdening the CPU and to fully exploit the power of current graphics hardware [16]. As a consequence, it is possible to employ commodity computers with much lower costs than would be necessary with the high-end, shared memory computers that have been normally used for the recreation of this type of environment.

These techniques are based on multiresolution models where the different levels of detail of the objects are grouped in a single data structure. Each time the virtual scene is drawn, the rendering program chooses the preferable level of detail depending on the position of the observer [21]. The terrain system is designed to perform asynchronous loading whenever possible to minimize the instantaneous drop in frame rate due to terrain loading.

A common approach for the visualization of large scale terrains consists of a multiresolution model for terrain texture which cooperates with a multiresolution model for terrain geometry [8]. In this approach, an image pyramid and a texture tree are constructed for each texture layer. Therefore, multiple texture layers can be associated with one ter- 
rain model and can be combined in different ways. Although new techniques that have been recently proposed for the planetary terrain visualization following this approach [13], these types of techniques demand a tightly coupled integration between both multiresolution models. Since MarsVR has been developed as multiplatform software and the implementations of the most common graphics intensive techniques depend on the hardware architecture, we have designed the terrain visualization system included in MarsVR using a decoupled mechanism. In this mechanism, we have divided the processing of terrain geometry and terrain texture. This division has been performed in order to maximize both the frame rate and the visual quality of the generated scenes, independently of the system platform

All real data used in the development of MarsVR have been obtained from the observations acquired by Mars Globa Surveyor (MGS) and Viking missions [18]. On the one hand, the data for generating the terrain geometry is based on topographical data obtained by the Mars Orbiter Laser Altimeter (MOLA) device. This device is included as an instrument aboard NASA's Mars Global Surveyor spacecraft. On the other hand, the data source for the terrain textures corresponds to the high resolution images obtained by the Imaging System included in Viking 2 Orbiter. The Mars Global Surveyor includes an image capturing system called MOC (Mars Orbiter Camera), but the available datasets obtained from this device offers a lower resolution than wholeplanet color map available from the Viking 2 mission.

\subsubsection{Processing the terrain geometry}

One of the main motivations behind the MarsVR project was the creation of an entertainment tool for educational purposes based on a three-dimensional model of the planet Mars. Since this tool was initially designed as a 3D tour (as it is described in Section 3.2), starting from an interplanetary flight and finishing as a virtual flight over the Mars surface, the range in the scale of the involved data was large. Like most of GIS applications, MarsVR handles geographic data ranging from local to global in scope [3, 13]. Therefore, an interface that easily handles this range in scale is important.

This interface has been defined as a globe-centric viewing environment. The nature of this Martian globe provides a seamless view of the planetary geography [6]. Figure 3 shows how this has been implemented at the lower resolution level of this interface. This level models the entire globe as a cube and divides it into six 90-degrees square planes. Four planes compose the central region of the globe, between 45 degrees north and south. The last two planes cover the polar regions. The surface geometry of the sphere has been augmented with elevation data.

The MOLA dataset (a packed set of data obtained from MOLA altimeter) is organized as a large two-dimensional matrix with longitude and latitude serving as the dimensions. The value at a given longitude and latitude is the average altitude for that area. This data is entered into a global grid where each point is spaced 60 kilometers (apart at the equator) and represents 27 million elevation measurements performed between 1998 and 1999. Each elevation point is known with an accuracy of 13 meters in general with large areas of the flat northern hemisphere with an accuracy of better than two meters.

Figure 3: Lower resolution levels of the proposed interface designed as a globe-centric environment and implemented using a six-sided cube.

The first step in the processing of the terrain geometry consisted in (after all radiometric and brightness corrections) the creation of a Digital Terrain Model (DTM) of the downloaded data. Despite this regular structure (composed of a huge amount of data) could be segmented into several square tiles, its processing would exceed the capacity of any computer (in terms of main memory and graphic card requirements). For this reason, we have decided to handle a terrain representation based on a quadtree approach.

A quadtree is a well-known structure in Computer Graphics $[6,8,16,20]$. Basically, a quadtree is a hierarchical data model that recursively decomposes a map into smaller regions. Each node in the tree has four children nodes, each of which represents one quarter of the area that the parent represents. Although several contributions which have been proposed both for real-time management of level-of-detail reduction and for display of highly complex polygonal surface data $[8,13,16]$, we have used the NRQT model [20]. The NRQT (Non-Restricted Quadtree Triangulation) model avoids the use of restricted quadtrees. A restricted quadtree is a limited quadtree whose neighboring elements must be the same size within a factor of two. The NRQT approach not only prevents possible cracks in the terrain surface, but also uses a lower number of triangles than restricted hierarchical triangulations, commonly used for this kind of purpose [16].

The off-line processing mechanism included in NRQT model considers a precomputation stage. In this stage, we obtained six levels of detail for each one of the six planes mentioned above. These six levels, corresponding to the six precalculated quadtree pyramids, generate 5461 nodes and require $2.79 \mathrm{~GB}$ of disk space. The content of each node represents the level of resolution for a different level of detail, the number of triangles included in these nodes ranges from 2 to 12608. The average number of triangles is approximately 1580 and the standard deviation is roughly 1933.

Figure 4 shows an example of a large scale Martian terrain visualization obtained from MarsVR (figure left) and the representation of this terrain using the non-restricted quadtree triangulation NRQT (figure right). The represented terrain corresponds to Olympus Mons considered as the largest known volcano of the solar system with a diam- 


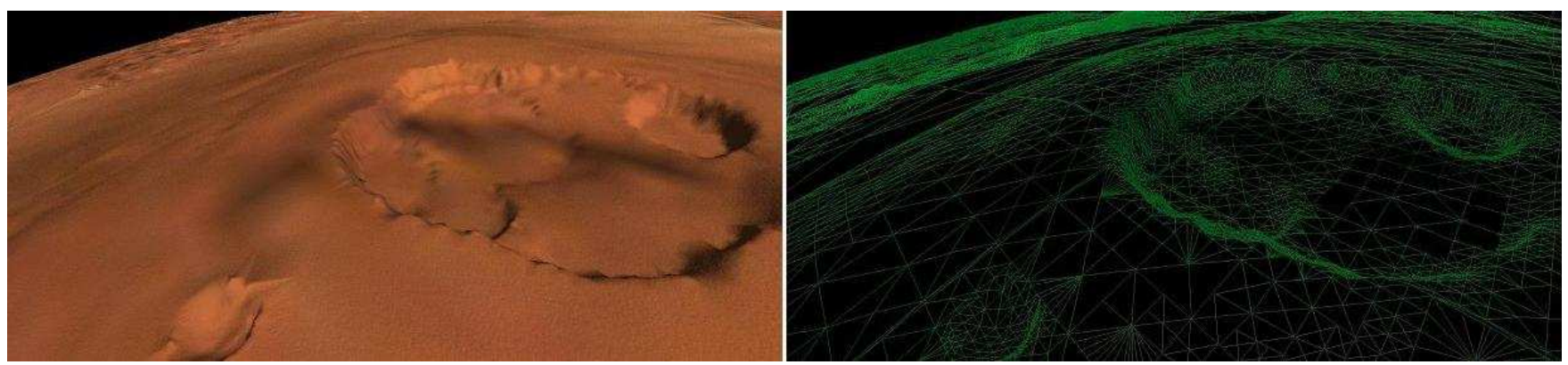

Figure 4: Images obtained from MarsVR corresponding to the Olympus Mons zone on Mars surface. Left: Final aspect of the terrain representation using the NRQT model. Right: Representation of the final triangle mesh based on the restricted quadtree representation of the space for the visualized terrain.

eter of 650 kilometers. Both images were obtained from an altitude of 35 kilometers. The right figure shows the node levels obtained from NRQT triangulations are independent from the rest. In this sense, and in order to keep continuity among neighboring regions with different resolution, this figure shows how triangle strips or triangle fans avoid cracks between neighboring tiles. Moreover, as any one other method based on quadtree representation of the terrain, the resolution of terrain tiles are constantly adapted according to the viewing distance.

\subsubsection{Processing the terrain textures}

There are several data sources containing thousands of pictures related to the surface of the planet Mars. However, most of them either show distorted pictures or do not cover the surface of the entire planet. The most appropriate image source for our purposes belongs to the United States Geographical Survey (USGS) Astrogeology Research Program. This organization has taken Viking 2 era photographs and processed them to eliminate spherical distortions and aligned the resulting photograph mosaics with landmarks in the MOLA data. The resulting dataset is called the Mars Global Digital Image Mosaic (MDIM) [1]. The current version of MDIM dataset (called MDIM 2.1) includes 30 files covering the planet, except for the poles. The total size of the files corresponding to this dataset version (256 pixels/degree version) is about 2.60GB.

Despite the quality of the images included, the MDIM dataset is four times higher than the MOLA data, the actual resolution of MDIM images is about 250 meters/pixel. Both the low resolution of the original textures and the huge size of the dataset have led us to incorporate some additional visualization techniques included in the current version of MarsVR. These techniques have allowed us to improve the visual quality of the generated virtual scenes and to manage this amount of texture data. As Section 3.1 describes, these advanced visualization techniques are specific to the graphics hardware and require an additional development effort when they are included as part of a multiplatform application. In order to cover the most important types of immersive visualization systems, we have addressed both problems from two different perspectives. On the one hand, techniques based on clipmapping textures give support to handle huge amount of textures. On the other hand, techniques based on vertex and pixel shaders address the problem of the low texture resolution by providing advanced lighting effects [9].

Clipmapping techniques were initially oriented to the graphics hardware included in most of Silicon Graphics Computers. A multiresolution image can be composed of different images that explicitly define the levels of details. Iris Performer, the Silicon Graphics standard, organizes texture in a pyramidal structure where each level has twice the resolution compared to the lower one [28]. The clipmapping technique determines which resolution is to be applied to each region depending on the view-point position. Following this technique, we have performed the construction of the clipmapping pyramid from the texture data included in MDIM dataset. Since this texture dataset can be joined as a single $32.768 \times 32.768$ texture, this large texture file was selected as the lower level of the clipmapping pyramid. Starting from this level, our design stacks eight texture levels. Taking into account that each level has half the resolution compared to the higher one, the top level of the clipmapping pyramid structure corresponds to the division of the Mars terrain into square sections of $512 \times 512$ pixels. The total clipmapping process takes an average of 50 minutes on a SGI Onyx2 workstation with 4 R10000 processors and 2 GB of RAM memory. In our case, this pyramid generation process generates 5461 texture files (to complete all texture levels) with a total size of about 4.00GB.

Although clipmapping techniques allow us to represent large terrains containing thousands of textures, the main problem in the visualization of Mars surface corresponds to the low resolution of the original textures. The lack of resolution provided by the MDIM dataset generates surfaces too smooth and creates very unrealistic terrains with low levels of roughness. In these cases, the reduced level of roughness can be increased by means of the combination of both vertex shader and pixel shader techniques. Vertex and Pixel shaders are currently available in the main of graphic card of the most common personal computers. Vertex and Pixel shaders are graphic functions that calculate the effects on a per-vertex or a per-pixel basis, respectively [9]. Among other purposes, these effects can provide a virtual roughness to surface without the need to model these depressions geometrically (bumpmapping effects). In our case, we have included a software implementation of bumpmapping effects based on a multitexture approach where a roughness texture map perturbs surface normals. 
Figure 5 shows the results of bumpmapping effects when they are applied to the recreation of virtual terrains in MarsVR system. Figure 5-left shows the direct visualization of the data included in MDIM texture dataset from an altitude of 32 kilometers. The represented zone corresponds to Valles Marineris, a system of troughs, chasms, and canyons that stretches more than 4000 kilometers across the Martian western hemisphere. This figure proves how the lack of texture resolution currently available in MDIM texture dataset generates low realistic terrain. Natural elements represented in the virtual terrain are too regular and lack roughness or deformation features. On the contrary, Figure 5-shows a more realistic appearance of the same terrain. In this case, the roughness texture map is designed on a $1024 \times 1024$ bumpmapping texture. The alteration of surface normal provided by this texture map generates a coarser surface terrain and generates a more realistic terrain visualization.

\section{DESCRIPTION OF THE EXPERIENCE}

In this section we present some of the results obtained in MarsVR. The results presented in this section are obtained in a multi-projection environment composed of three projection surfaces. These mobile immersive visualization systems are very common in rotating temporary attractions or exhibitions that are called C3 systems or BabyCAVEs [7, 12, 29].

Our C3 system provides active stereoscopic visualization and is operated by $6 \mathrm{PCs}$ where each of them is an Intel Pentium-D Dual Core $2.8 \mathrm{GHz}$ with 2 GB RAM. All computers are interconnected through a Cisco Catalyst 3750 Gigabit Ethernet switch. One of the computers is configured as master and manages 5 slave PCs, each of them controls one of 6 projectors. These 6 projectors generate stereo images on the 3 walls of the CAVE. These walls compose a 160-degree panoramic wide cylindrical screen. All computers are equipped with nVidia GeForce FX 6200 graphics card with $512 \mathrm{MB}$ of DDR2 graphics memory. Despite some high-level libraries include clustering computing for graphics purpose[4], the management of client/server communications has been included as a internal module in the architecture design of MarsVR. These design decisions have provided us with a complete solution, fully developed on OpenGL Performer 3.0.

Figure 6, Figure 7 and Figure 8 show some screenshots of the main stages included in MarsVR. Since MarsVR places users into an interactive 3D tour through the planet Mars, this tour has been divided into four interactive areas. The transition among these areas is controlled by the finite state machine described in Section 3.2. Figure 6 shows the beginning of the $3 \mathrm{D}$ tour where the user is involved in an interplanetary spaceflight of about two minutes of duration. This interplanetary spaceflight is launched from the Kennedy Space Center in Port Canaveral (Florida, USA) heading to the Diacria region, located in the northern hemisphere of the planet Mars. During the course of the spaceflight, users not only pass by the International Space Station (Figure 6-up), but also they realize the existing distance between planets in the Milky Way (Figure 6-down).

Once the spaceflight is approaching Mars, the spacecraft changes the trajectory and starts a circular route about the planet. The average altitude of this circular flight around the Martian surface is about 25 kilometers. Since users can change the speed flight (using a joystick located in the first

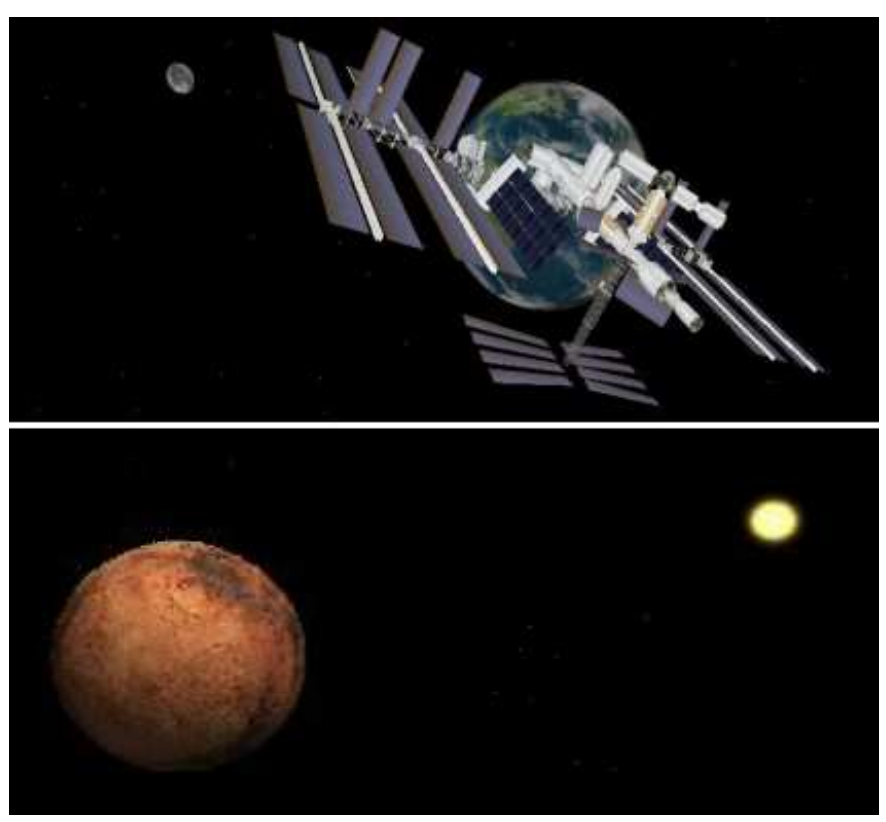

Figure 6: Snapshots obtained from the first stage of the 3D tour. Up: Approach maneuver close to the International Space Station. Down: A view of Mars and the Sun.

seat rows of the BabyCAVE) ranging from about $20 \mathrm{~km} / \mathrm{s}$ to $250 \mathrm{~km} / \mathrm{s}$, this circular flight allows users two explore the most important regions of the planet Mars in few minutes. Figure 4 and Figure 5 show some snapshots from this flight, Figure 7 shows new images obtained from other executions of this virtual trip. In this case, Figure 7-up shows an image captured in the Olympus Mons zone at an altitude of 4.60 kilometers. Figure 7-down shows a snapshot image of the Moeris Lacus region. Moeris Lacus area is a well-know small protrusion entirely degraded by wind erosion. This image was obtained at an altitude of 9.28 kilometers above the surface level.

MarsVR limits the duration of the spaceflight around the surface of the planet Mars. The maximum duration of the flight, which speed can controlled by the user, is about twelve minutes. Figure 8-up depicts what happens when the maximum flight time is reached. In this moment, the system takes control of the simulation automatically and forces the landing of the spacecraft.

The automatic landing is performed on a land station located at the Noachis region (southern hemisphere). Once the landing process is completed, MarsVR starts the last stage of the $3 \mathrm{D}$ tour. In this stage, the user is introduced into a roller coaster ride. This roller coaster ride takes about two minutes and is performed on a dynamic railway route. Therefore, the total maximum duration for the whole experience including the interplanetary flight, the martial surface flight and the roller coaster ride is about fourteen minutes. We have stated our railway route is dynamic because part of its geometry is randomly generated in each execution of MarsVR. The railway route includes a 360-degree high speed spiral, two double loops and several corkscrew turns for all 


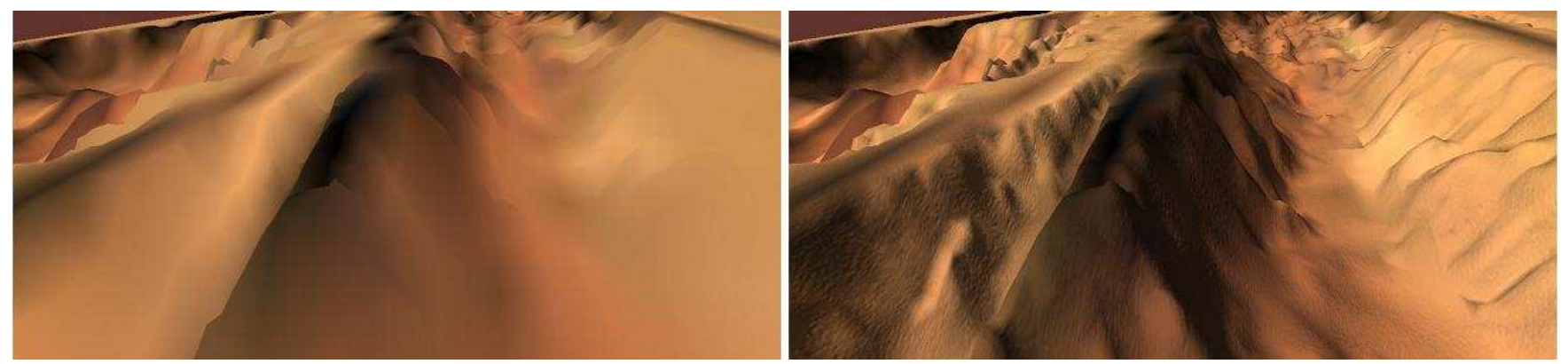

Figure 5: Images obtained from MarsVR corresponding to the Valles Marineris zone on Mars surface. Left: Direct visualization of Martian surface without preprocessing the texture data included in MDIM dataset. Right: Final aspect of the terrain representation using a bumpmapping technique implemented using shaders.
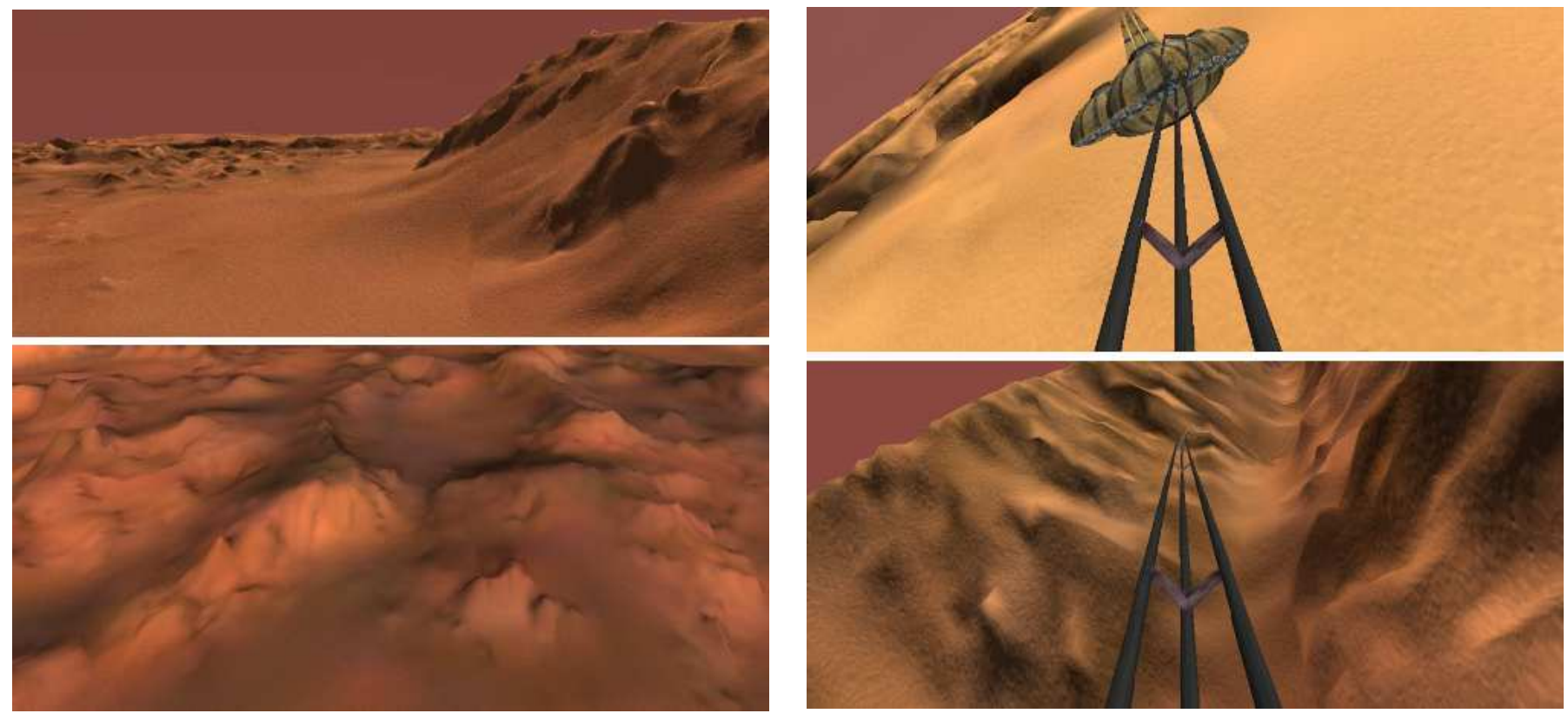

Figure 7: Some images from the spaceflight. Up: Olympus Mons zone at an altitude of 4.60 kilometers. Down: Moeris Lacus area at an altitude of 9.28 kilometers

Figure 8: Images of the last stage of the $3 \mathrm{D}$ virtual tour. Up: Automatic landing maneuver on a land station located at the Noachis region. Down: Roller coaster ride on the dynamic railway route 
the feasible cases. Figure 8-down shows a snapshot of this out-of-the-ordinary ride.

Finally, Figure 9 shows a picture of the final system exhibited with much success at the Science Museum of Valencia, in Spain (October, 2005). A very similar configuration of the system was also shown at the Science Exhibition in Granada (Spain) a few months later (February, 2006). This picture depicts the execution of MarsVR on a mobile immersive visualization system similar to the one described above. When this image was taken users were in the spaceflight stage of the 3D tour. In that moment, information obtained from HUD panel (located in the top right corner of the most to the right screen) showed they were flying over Valles Marineris area at an altitude of 94.76 kilometers with a speed of $127 \mathrm{~km} / \mathrm{s}$.

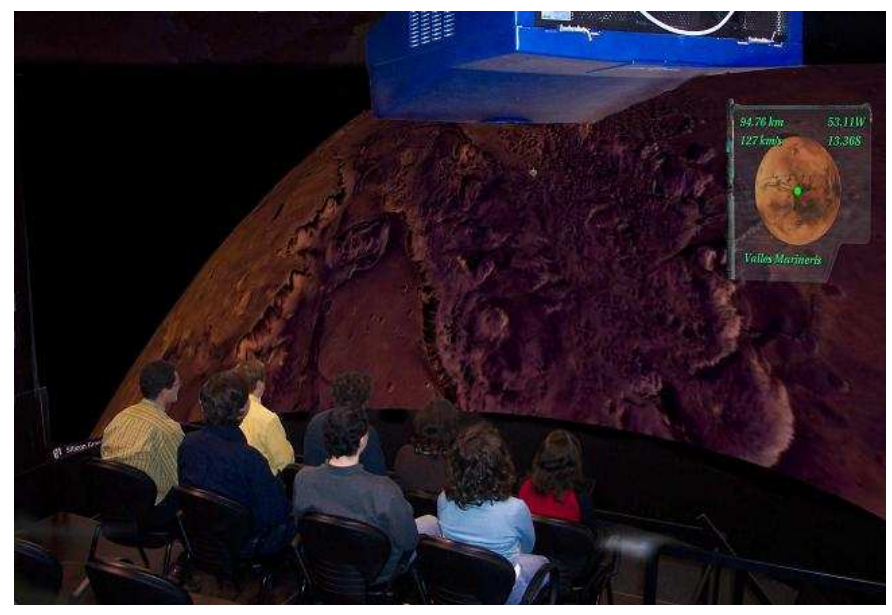

Figure 9: A image of MarsVR on the mobile immersive visualization system exposed at the Science Museum of Valencia (Spain) in October 2005

\section{CONCLUSIONS AND FUTURE WORK}

MarsVR is a virtual reality entertainment application for educational purposes centered on the simulation of spaceflights over the Mars surface. The basic idea of MarsVR is to reach the general public interested in astronomy and space exploration and have not had the opportunity to use immersive visualization devices for exploring amazing extraterrestrial landscapes. Although other interactive applications based on virtual reality technologies have achieved very realistic simulations of the Martian surface, all of them have been developed for scientific purposes.

The data collection included in MarsVR has been obtained from the main references in the fields of astronomy and astrophysics. In order to efficiently handle this amount of data, while offering a high level of visual quality, MarsVR incorporates some of the latest techniques in the field of 3D real time graphics. In this sense, a multiresolution model of the terrain consisting in a new hierarchial triangulation based on a non-restricted quadtree representation is included. Moreover, bumpmapping effects based on vertex and pixel shaders provide virtual roughness to Mars surface.

Nowadays, MarsVR has been presented in some science exhibitions. The results show that MarsVR can be consid- ered as an entertaining interactive application for navigating over the Mars surface, as well as providing very useful and real information on several aspects of this planet.

As future work continues, we plan to implement a new version of the module for processing texture terrain. Since new high-resolution textures obtained from Mars Orbital Camera will become available early 2008, our idea consists in performing an automatic terrain texture processing independent of texture resolution.

\section{REFERENCES}

[1] B. Archinal, R. Kirk, T. Duxbury, E. Lee, R. Sucharski, and D. Cook. Mars Digital Image Model (MDIM) 2.1 control network. In Proceedings of ISPRS Working Group IV/9 Workshop Advances in Planetary Mapping 2003. International Society for Optical Engineering, March 2003.

[2] J. Bates. Virtual Reality, Art and Entertainment. PRESENCE: Teleoperators and Virtual Environments, 1(1), pages 133-138, 1992.

[3] B. Beeson, M. Lancaster, D. Barnes, P. Bourke, and G. Rixon. Visualising Astronomical Data using VRML. In Proceedings of the SPIE Digital Library, volume 5493, pages 242-253. International Society for Optical Engineering, March 2004.

[4] A. Bierbaum, C. Just, P. Hartling, K. Meinert, A. Baker, and C. Cruz-Neira. VR Juggler: A Virtual Platform for Virtual Reality Application Development. In Proceedings of the IEEE Virtual Reality, pages 89-96. IEEE Society Press, March 2001.

[5] R. Burden and J. Faires. Numerical Analysis. Pacific Grove, CA: Brooks/Cole, 7th ed., 2001.

[6] C. Crawford, S. Bayarri, and D. Petrovic. Fast 3D Visualization of Large Image Datasets in a GIS. Earth Observation Magazine, 12(9), pages 12-17, December 2003.

[7] C. Cruz-Neira, D. Sandin, and T. DeFanti. Surround-Screen Projection-Based Virtual Reality: The Design and Implementation of the CAVE. In Proceedings of the ACM SIGGRAPH Computer Graphics Conference, pages 135-142. ACM Press, September 1993.

[8] J. Dollner, K. Baumann, and K. Hinrichs. Texturing techniques for terrain visualization. In Proceedings of the 11th IEEE Visualization Conference (VIS 2000), pages 227-234. IEEE Society Press, October 2000.

[9] W. Engel. Programming Vertex and Pixel Shaders (Programming Series). Charles River Media, September 2004.

[10] A. Forsberg, G. Morgan, N. Petro, J. Levy, and J. Head. Virtual Field Trip to Mars: Experiences with a Virtual Reality Lab for Undergraduate student. In Proceedings 37th Lunar and Planetary Science Conference (LPSC'2006), pages 1319-1320. National Aeronautics and Space Administration (NASA), March 2006.

[11] G. Gurzadyan. Theory of Interplanetary Flights. Taylor and Francis, 1996.

[12] J. Head, A. van Dam, S. Fulcomer, A. Forsberg, and G. R. adn S. Milkovich. ADVISER: Immersive Scientic Visualization Applied to Mars Research and Exploration. Photogrammetric Engineering and Remote Sensing, 71(10), pages 1219-1226, October 2005.

[13] L. Hwa, M. Duchaineau, and K. Joy. Real-time optimal adaptation for planetary geometry and texture: 4-8 tile hierarchies. IEEE Transactions on Visualization and Computer Graphics, 11(4), pages 355-368, July 2005.

[14] D. Kushner. Masters of Doom: How Two Guys Created an Empire and Transformed Pop Culture. Random Houses, 2003.

[15] B. Laurel, R. Strickland, and R. Tow. Placeholder: Landscape and Narrative In Virtual Environments. ACM Computer Graphics Quarterly, 28(2), pages 121-132, May 1994. 
[16] P. Lindstrom and V. Pascucci. Terrain simplification simplified: A general framework for view-dependent out-of-core visualization. IEEE Transactions on Visualization and Computer Graphics, 8(3), pages 239-254, July 2002.

[17] M. McGreevy. Virtual Reality and Planetary Exploration, pages 163-197. Academic Press, 1993.

[18] W. Newcott. Return to Mars. National Geographic, volume 194, pages 2-29, 1998.

[19] P. Peggy-Li and D. Curkendall. Parallel Three Dimensional Perspective Rendering. In Proceedings of European Workshop on Parallel Computing. From Theory to Sound Practice (EWPC'92), pages 320-331. IOS Press, March 1992.

[20] M. Perez, R. Olanda, and M. Fernandez. Visualization of Large Terrain Using Non-restricted Quadtree Triangulations. In Proceedings of the International Conference in Computational Science and Its Applications (ICCSA'2004) -Lecture Notes in Computer Science, volume 3044, pages 671-681, 2004.

[21] T. Polack. Focus On 3D Terrain Programming (Game Development). Course Technology PTR, December 2002.

[22] M. Roth, G. Vo, and D. Reiners. Multi-threading and Clustering for Scene Graph Systems. Computer and Graphics, volume 26(1), pages 63-66, February 2004.

[23] M. Schuemie, M. Bruynzeel, L. Drost, M. Brinckman, G. de Haan, P. Emmelkamp, and C. van der Mast. Treatment of Acrophobia in Virtual Reality: a Pilot Study. In Proceedings of Conference Euromedia 2000, pages 271-275. Euromedia Group, May 2000.

[24] F. Seron, D. Gutierrez, G. Gutierrez, and E. Cerezo. Visualizing Sunsets through Inhomogeneous Atmospheres. In Proceedings of Computer Graphics International 2004 (CGI'04), pages 349-56. IEEE Society Press, June 2004.

[25] O. Staadt, J. Walker, C. Nuber, and B. Hamann. A Survey and Performance Analysis of Software Platforms for Interactive Cluster-Based Multi-Screen Rendering. In Proceedings of the Workshop on Virtual Environments 2003, pages 261-270. ACM Press, June 2003.
[26] C. Stoker, T. Blackmon, J. Hagen, B. Kanefsky, D. Rasmussen, K. Schwehr, M. Sims, and E. Zbinden. Marsmap: An Interactive Virtual Reality Model of the Pathfinder Landing Site. In Proceedings of Lunar and Planetary Science Conference (LPSC'98), pages CD-ROM. Lunar and Planetary Institute, March 1998.

[27] C. Stoker, E. Zbinden, T. Blackmon, and L. Nguyen. Visualizing Mars Using Virtual Reality: A State of the Art Mapping Technique Used on Mars Pathfinder. In Proceedings of the 5th Internation Conference of Mars. Lunar and Planetary Institute, March 1999.

[28] C. Tanner, C. Migdal, and M. Jones. The Clipmap: A Virtual Mipmap. In Proceedings of the ACM SIGGRAPH Computer Graphics Conference. ACM Press, August 1998.

[29] A. Van Dam, D. Laidlaw, and R. Simpson. Experiments in immersive virtual reality for scientific visualization. Computer and Graphics, 26, pages 535-555, 2002.

[30] S. Walker and J. Kenneth. Large haptic topographic maps: marsview and the proxy graph algorithm. In Proceedings of the ACM SIGGRAPH 2003. Symposium on Interactive 3D Graphics, pages 83-92. ACM Press, April 2003.

[31] S. Wang, M. Damon, and D. Yuen. Visualization in the Earth Sciences: A Discussion on Various Visualization Methods using Amira. In Proceedings of the 16th IEEE Visualization Conference (VIS 2005), page 112. IEEE Society Press, October 2005.

[32] Z. Wartell, W. Ribarsky, and L. Hodges. Third-person navigation of whole-planet terrain in a head-tracked stereoscopic environment. In Proceedings of the IEEE Virtual Reality Conference, pages 141-149. IEEE Society Press, March 1999.

[33] T. Wayne. Roller coaster physics. Wayne, 1998.

[34] G. Wong and V. Wong. Virtual Reality in Space Exploration. Surveys and Presentations in Information Systems Engineering (SURPRISE), 4, 1996.

[35] Y. Yair, R. Mintz, and S. Litvak. 3D-virtual Reality in Science Education: An Implication for Astronomy Teaching. Journal of Computers in Mathematics and Science Teaching, 20(3):294, 2001.

[36] M. Zyda. From visual simulation to virtual reality to games. IEEE Computer, volume 38(9), pages 25-32, September 2005. 